\title{
Early Prediction of Tumor Response to Bevacizumab Treatment in Murine Colon Cancer Models using Three-Dimensional Dynamic Contrast-Enhanced Ultrasound Imaging
}

\author{
Jianhua Zhou ${ }^{1,2}$, Huiping Zhang ${ }^{1}$, Huaijun Wang ${ }^{1}$, Amelie M Lutz ${ }^{1}$, Ahmed El Kaffas ${ }^{1}$, Lu \\ Tian $^{3}$, Dimitre Hristov ${ }^{4}$, and Jürgen K. Willmann ${ }^{1}$ \\ ${ }^{1}$ Department of Radiology, Molecular Imaging Program at Stanford, Stanford University, School of \\ Medicine, Stanford, California, USA \\ ${ }^{2}$ Department of Ultrasound, Sun Yat-Sen University Cancer Center, State Key Laboratory of \\ Oncology in South China, Collaborative Innovation Center for Cancer Medicine, Guangzhou, \\ China \\ ${ }^{3}$ Department of Health, Research \& Policy, Stanford University, Stanford, California, USA \\ ${ }^{4}$ Department of Radiation Oncology, Stanford University, Stanford, California, USA
}

\section{Abstract}

\begin{abstract}
Due to spatial tumor heterogeneity and consecutive sampling errors, it is critically important to assess treatment response following antiangiogenic therapy in three dimensions as twodimensional assessment has been shown to substantially over- and underestimate treatment response. In this study, we evaluated whether three-dimensional (3D) dynamic contrast-enhanced ultrasound (DCE-US) imaging allows assessing early changes in tumor perfusion following antiangiogenic treatment (Bevacizumab administered at a dose of $10 \mathrm{mg} / \mathrm{kg} \mathrm{b.w.),} \mathrm{and} \mathrm{whether}$ these changes could predict treatment response in colon cancer tumors that either are responsive (LS174T tumors) or none responsive (CT26) to the propose treatment. Our results showed that the perfusion parameters of 3D DCE-US including peak enhancement (PE) and area under curve (AUC) significantly decreased by up to 69\% and 77\%, respectively, in LS174T tumors within 1 day after antiangiogenic treatment $(P=0.005)$, but not in CT26 tumors $(P>0.05)$. Similarly, the percentage area of neovasculature significantly decreased in treated vs. control LS174T tumors $(P<0.001)$, but not in treated vs. control CT26 tumors $(P=0.796)$. Early decrease in both PE and AUC by $45-50 \%$ were predictive of treatment response in $100 \%$ (95\% CI, $69.2 \%, 100 \%$ ) of responding tumors, and in $100 \%$ (95\% CI, 88.4\%, 100\%) and 86.7\% (95\% CI, 69.3\%, 96.2\%), respectively, of nonresponding tumors. In conclusion, 3D DCE-US provides clinically relevant information on the variability of tumor response to antiangiogenic therapy and may be further developed as biomarker for predicting treatment outcomes.
\end{abstract}

Address all correspondence to: Jürgen K. Willmann, M.D., Department of Radiology, Molecular Imaging Program at Stanford, School of Medicine, Stanford University, 300 Pasteur Drive, Room H1307; Stanford, CA 94305-5621, P: 650-723-5424; Fax: 650-723-1909, willmann@stanford.edu.

Conflict of Interest:

There is no conflict of interest. 


\section{Keywords}

three-dimensional dynamic contrast-enhanced ultrasound; antiangiogenic therapy; microbubbles; colon cancer

\section{Introduction}

Angiogenesis, the formation of new capillary blood vessels from pre-existing vasculature, is necessary for tumor growth beyond 1 to $2 \mathrm{~mm}$ in diameter [1]. Given the critical role of angiogenesis in tumor growth, progression, and metastasis [2], several antiangiogenic therapies have already been approved for clinical use [3]. Combination treatments where antiangiogenic agents such as bevacizumab (a humanized anti-VEGF monoclonal antibody) are given in conjunction with chemotherapy have resulted in improved progression-free survival and overall survival in patients with metastatic colon cancer [4,5].

Treatment response in clinical cancer trials is conventionally assessed using the Response Evaluation Criteria in Solid Tumor (RECIST) method, which is based on changes in maximum tumor diameters [6]. However, antiangiogenic agents result in acute cytostatic effects that diminish tumor blood supply, while often minimally affecting tumor size [7]. Therefore, quantifying post treatment changes based on RECIST may not reflect tumor response, in particular at early time points. To minimize unnecessary cost and side effects in nonresponding patients, alternative methods or tools are needed to allow rapid assessment of treatment effects within the first days/weeks of treatment. Early changes in functional or molecular imaging-based biomarkers could provide such a method, and could be used to predict treatment response, thereby resulting in improved cancer patient management and personalized treatments.

Several imaging approaches such as positron emission tomography (PET), dynamic contrastenhanced magnetic resonance imaging (DCE-MRI), dynamic contrast-enhanced computed tomography (DCE-CT), and dynamic contrast-enhanced ultrasound (DCE-US) are being considered for monitoring of tumor response to novel molecularly-targeted therapies such as antiangiogenic drugs [8]. While ultrasound currently does not play a significant clinical role in oncological imaging since it is not a whole body exam which is needed for traditional tumor response assessment usually several months into the treatments, it may be an attractive technique for early response assessments of target lesions that are accessible for ultrasound, such as metastases in the liver. Unlike other imaging modalities, ultrasound does not expose patients to radiation, is relatively inexpensive, is portable, and is globally available for bedside imaging. Recent commercial availability of three-dimensional (3D) DCE-US has enabled more reliably and accurate quantification of tumor blood perfusion by eliminating 2D sampling errors that can occur in longitudinal consecutive imaging applications such as treatment monitoring [9-11]. Tumor imaging is particularly susceptible to sampling errors due to spatial vascular heterogeneities linked to a hypoxic and necrotic microenvironment [9].

The purpose of this study was to evaluate whether 3D DCE-US imaging allows assessing early changes in tumor perfusion following antiangiogenic treatment and whether these 
changes could predict treatment response in two animal models of colon cancer that simulate clinical responders and nonresponders.

\section{Materials and Methods}

\section{Subcutaneous Colon Cancer Xenografts in Mice and Antiangiogenic Treatment}

All experimental procedures involving laboratory animals were approved by the Institutional Animal Care and Use Committee. Forty female nude mice (Charles River, Wilmington, MA; 6-8 weeks old; 20-25 g) were used for inducing the colon cancer models. The human colon cancer cell line LS174T (ATCC, Manassas, VA), which has been shown to be sensitive to the treatment of VEGF pathway blockade [12], was used to simulate clinical responders, while the murine colon cancer cell line CT26.WT (ATCC, Manassas, VA), which has been shown to be resistant to VEGF pathway blockade [13,14], was used to simulate clinical nonresponders. Both cell lines were grown in Dulbecco's Modified Eagle Medium (DMEM; Gibco, Grand Island, NY), supplemented with $10 \%$ fetal bovine serum (Gibco), penicillin $(50 \mathrm{U} / \mathrm{ml})$, and streptomycin $(50 \mu \mathrm{g} / \mathrm{ml})$ at $37^{\circ} \mathrm{C}$ in a humidified $5 \% \mathrm{CO} 2$ atmosphere. At $70 \%-80 \%$ confluence, the tumor cells were collected following trypsinization, and $4 \times 10^{6}$

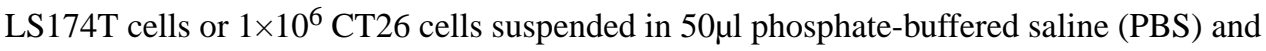
50 $\mu$ l Matrigel (BD Biosciences, San Jose, CA) were injected subcutaneously on the right lower hind limb of all nude mice. Both LS174T and CT26 tumors were allowed to grow for 10 days after cancer cell injection to reach 6-15 mm (mean, $10 \mathrm{~mm}$ ) in maximum diameter, which was measured by using an electronic caliper available on the ultrasound system. The average tumor volume doubling time for LS174T colon cancer tumors was 3.7 days and 3 days for CT26 tumors. Mice with either tumor type were randomized into 1) the treatment group ( $\mathrm{n}=10$ from each cell type; total, 20 tumors); and 2) the control group ( $\mathrm{n}=10$ each; total, 20 tumors). The clinically used antiangiogenic agent bevacizumab (Avastin, Genentech, South San Francisco, CA) diluted in sterile saline was administered by means of intravenous injection on days 0 (baseline), 3 , and 7 at a dose of $10 \mathrm{mg} / \mathrm{kg} \mathrm{b.w}$. (corresponding to a fluid volume of $10 \mu \mathrm{l} / \mathrm{g}$ b.w.) for animals in the treatment group (including responders and nonresponders). Since tumors in nude mice grow faster than those in patients, in general bevacizumab dosing regimen is different in mice than in humans. The dosing regimen used in this study was based on previous animal studies [15-18]. Mice in the control group received the same volume of sterile saline only with the same timing and dosing schedule used for the treatment group. (Figure 1).

\section{Monitoring Antiangiogenic Treatment with 3D DCE-US Imaging}

For all imaging procedures, mice were anesthetized with inhalation of $2 \%$ isoflurane in room air (administered at $2 \mathrm{~L} / \mathrm{min}$ ). Mice were placed prone on a heated imaging stage in order to maintain constant body temperature for the entire duration of the experiments. A 27G needle catheter (Vevo Micromarker; VisualSonics, Toronto, Canada), which attached to an infusion pump (Kent Scientific, Torrington, CT), was placed into one of the two tail veins for contrast agent injection. Successful placement was confirmed before contrast agent injection each time by observing blood filling into the catheter tube. All animals were imaged with 3D DCE-US imaging on days $0,1,3,7$, and 10 using a clinical ultrasound scanner (EPIQ 7; Philips Healthcare, Andover, MA) coupled to a clinical matrix array 
transducer (X6-1; Philips). The transducer was held in a fixed position with a clamp to minimize motion artifacts. To reduce artifacts in the near-field zone of the clinical transducer, a customized standoff ultrasound gel was placed on the skin of all mice. The distance between the transducer and the center of the tumor was set at $3 \mathrm{~cm}$. In all tumors, 3D DCE-US imaging was performed in power modulation contrast imaging mode using the following imaging setting: center frequency, 3.2 MHz; mechanical index, 0.09; volume rate, $1 \mathrm{~Hz}$; dynamic range, $52 \mathrm{~dB}$; focus length, $5 \mathrm{~cm}$. Tumor growth was monitored at each time by using electronic calipers measurements available on the ultrasound system. The greatest longitudinal, transverse, and anteroposterior dimensions of each tumor were measured in grayscale imaging. Tumor volumes were determined by using the following formula: volume $=\pi / 6 \times L \times W \times H$, where $L$ is length, $W$ is width, and $H$ is height.

All ultrasound examinations were performed by one radiologist (with 10 years experience in contrast-enhanced ultrasound), who was blind to the treatment status. Three dimensional DCE-US imaging data sets were acquired by using the bolus technique which is based on the wash-in and wash-out kinetics of contrast microbubbles after a single intravenous bolus injection [19]. FDA-approved perflutren microbubbles (Definity; Lantheus Medical Imaging, Inc., North Billerica, MA) were used (diluted 1:4 in sterile 0.9\% saline solution after activation) and a $120-\mu \mathrm{L}$ suspension was administered within a 5 -second bolus (at a constant injection rate of $24 \mu \mathrm{l} / \mathrm{sec}$ ) by using the infusion pump. Three-dimensional DCE-US datasets were acquired in real time continuously for four minutes in each mouse through a built-in Digital Navigation Link connecting the ultrasound system directly to custom inhouse MevisLab modules (MeVis Medical Solutions AG, Bremen, Germany) written in C++ [20].

\section{Image Analysis of 3D DCE-US Data Sets}

Image analysis was performed by one reader with 6 years of DCE-US experience in random order. The reader was blinded to the treatment information and types of animal (responder vs. nonresponder). All 3D DCE-US imaging datasets were analyzed with a custom software which was developed by using the software package MeVisLab [21]. A volume of interest (VOI) was manually contoured covering the whole tumor visualized on sagittal, longitudinal, and coronal planes. Voxel intensities were then linearized to obtain an approximate linear depiction of the backscattered signal intensity, and a time-intensity curve (TIC) was obtained from the average voxel intensities within the VOI. A first-pass kinetics analysis was performed to obtain perfusion parameters by fitting a lognormal perfusion model to the TIC.[19,22]. The following perfusion parameters were calculated as previously described $[9,23,24]$ from the fitted TIC [22]: peak enhancement (PE, arbitrary units, a.u.), area under the curve (AUC, a.u.), and time to peak (TTP, seconds). PE was defined as the maximum increase in the signal intensity following bolus injection of the contrast agent. AUC was defined as the total area under the curve of the fitted TIC. Both PE and AUC are proportional to blood volume. TTP was defined as the time interval from the beginning of enhancement to the peak of the fitted curve, and is generally related to blood flow. Tumor volumes and all 3D DCE-US perfusion parameters after treatment were normalized to the baseline values to show percent changes. 


\section{Ex Vivo Analysis of the Tumor Neovasculature}

At day 10, all mice were euthanized after the imaging exam, and tumors were surgically removed and fixed in $4 \%$ paraformaldehyde and PBS solution for $24 \mathrm{~h}$. Tumors were then cryopreserved in a 30\% sucrose and PBS solution (Sigma Aldrich, St Louis, Mo). Tissue samples were placed in optimum cutting temperature (OCT, Fisher Scientific, Pittsburgh, $\mathrm{Pa}$ ), and then sectioned into $10 \mu \mathrm{m}$ slices for immunofluorescence staining. For neovasculature analysis, slices were stained for the vascular endothelial marker CD31. Standard methods were used for immunofluorescence staining [25]. Briefly, tumor slices were rinsed with PBS for 10 minutes to remove remaining OCT and permeabilized in $0.5 \%$ Triton-X 100 for 10 minutes. Tissue sections were blocked in a solution that contained 3\% goat serum (Sigma, St Louis, MO), 3\% bovine serum albumin (Sigma, St Louis, MO), and $3 \%$ donkey serum (Sigma, St Louis, MO) for up to $5 \mathrm{~h}$ at room temperature to remove nonspecific staining. A rat anti-mouse CD31 antibody, with 1:100 dilution (eBioscience, San Jose, CA), was used as primary antibody to incubate the tumor specimens overnight at $4{ }^{\circ} \mathrm{C}$. After rinsing with PBS, a secondary antibody (1:250 AlexaFluor488 donkey-anti-rat IgG; Invitrogen, Grand Island, NY) was added to make the primary antibody visible. Samples were mounted by using ProLong Gold (BiogeneX, San Ramon, CA). Fluorescent microscopy was performed by using a LSM510 metaconfocal microscope (Zeiss, Maple Grove, MN) and a high-resolution digital camera (AxioCam MRc, Bernried, Germany) under 200-fold magnification. The percentage area of neovasculature per slice was quantified by using Image $\mathbf{J}$ software (National Institutes of Health, Bethesda, MD) as the average value from 5 randomly selected fields of view (single field of view area, $0.19 \mathrm{~mm}^{2}$ ).

\section{Statistical analysis}

The nonparametric paired-samples Wilcoxon rank test was used to compare the changes in tumor volume and perfusion parameters (PE, AUC and TTP) following treatment. The nonparametric Mann-Whitney $U$ test was used to assess the statistical significance in tumor volume, perfusion parameters, and ex vivo percentage area of neovasculature between treated and control groups. To assess whether relative changes in the perfusion parameters of 3D DCE-US at day 1 compared to baseline could predict treatment response at a later time point (day 10), perfusion parameters were compared to the relative change of tumor volume at day 10 with tumor progression defined as a 4 -fold or more increase in tumor volume from day 0 to day 10 . The $95 \%$ confidence intervals (CI) were constructed with exact method due to small sample sizes. A $P$ value of $<0.05$ was considered statistically significant. All analyses were performed by using IBM SPSS statistics software version 22 (IBM Corp, Chicago, IL).

\section{Results}

\section{Antiangiogenic therapeutic effects on tumor growth}

No significant differences were noted between control and treated tumors at day 0 for both the responder (LS174T, $P=0.247)$ and nonresponder (CT26, $P=0.218)$ groups. For the responder group, the tumor volume of saline-treated mice increased significantly (by 543\%; $P=0.005$ ) when compared to bevacizumab-treated mice (by $83 \% ; P=0.005$ ). On days 3,7 , and 10 , the relative increase in tumor volume $(P<0.001)$ in bevacizumab-treated mice was 
significantly smaller than saline-treated mice; no significant difference was observed at day

$1(P=0.631)$.

In contrast, in the nonresponder group, tumor volumes in both bevacizumab-treated (by $887 \%$ ) and saline-treated (by 990\%) mice significantly increased $(P<0.05)$ at 1, 3, 7 and 10 days. There were no significant differences in relative increases of tumor volumes compared to baseline between bevacizumab-treated and saline-treated nonresponding tumors at any time point ( $P$ from 0.280 to 0.912 ) (Figure 2).

\section{Assessment of tumor perfusion with 3D DCE-US}

Longitudinal changes in 3D DCE-US perfusion parameters after bevacizumab treatment are summarized in Figure 3. In the responder group, bevacizumab-treated tumors showed a significant decrease in the 3D DCE-US perfusion parameters PE and AUC on all days as compared with day $0(P=0.005)$, while saline-treated tumors had no significant change in AUC at any time point ( $P$ from 0.153 to 0.878 ) and in PE at the first three time points ( $P$ from 0.102 to 0.540$)$; PE significantly decreased at day $10(P=0.005)$, possibly due to tumor necrosis. Overall, relative changes in PE and AUC were significantly decreased in bevacizumab-treated tumors as compared with saline-treated tumors on days 1, 3, 7, and 10 $(P<0.05)$. There were no significant differences in TTP in bevacizumab- or saline-treated mice at any time point ( $P$ from 0.393 to 0.912 ).

In contrast, in the nonresponder group, no significant differences in relative changes of all 3D DCE-US perfusion parameters were observed (PE, AUC, and TTP) in bevacizumabversus saline-treated tumors at any time point (for PE: $P$ ranged from 0.280 to 0.912 ; for AUC: $P$ from 0.075 to 1.000 ; for TTP: $P$ from 0.089 to 0.796 ) (Figure 4).

\section{Prediction of treatment response}

When AUC decreased by $45 \%$ or more at day 1 following initiation of bevacizumab treatment, treatment response could be predicted in $100 \%$ (95\% CI; $69.2 \%, 100 \%)$ of responding and in $86.7 \%(95 \% \mathrm{CI} ; 69.3 \%, 96.2 \%)$ of nonresponding tumors. All tumors $(95 \%$ CI; $86.8 \%, 100 \%)$ progressed by day 10 when AUC decreased by less than $45 \%$ at day 1.

At a 50\% or more decrease of PE at 1 day following initiation of bevacizumab treatment, a treatment response could be predicted in 100\% both in responding (95\% CI; 69.2\%, 100\%) and nonresponding (95\% CI; 88.4\%, 100\%) tumors. All tumors with a less than $50 \%$ decrease in PE progressed (95\% CI; 88.4\%, 100.0\%).

\section{Ex vivo quantitative immunofluorescence analysis of tumor neovasculature}

In responding tumors, the percentage area of neovasculature was significantly $(P<0.001)$ decreased in bevacizumab-treated $(1.04 \% \pm 0.43 \%)$ vs. saline-treated tumors $(4.95 \% \pm 0.35 \%)$. In contrast, in nonresponding tumors, there were no significant differences $(P=0.796)$ in the percentage area of neovasculature between bevacizumab-treated $(5.05 \% \pm 0.64 \%)$ and salinetreated tumors $(4.96 \% \pm 0.63 \%$; Figure 5). 


\section{Discussion}

In this study, the 3D DCE-US perfusion parameters PE and AUC were found sensitive to longitudinal treatment response following antiangiogenic therapy in a mouse model simulating clinical responders and nonresponders. In responding tumors, PE and AUC substantially decreased within 1 day following a single dose of bevacizumab, whereas no significant changes in PE and AUC were noted at any time point in the nonresponding tumors. Tumor responds to antiangiogenic therapy was further confirmed by tumor volume assessed at the end of the treatment. In addition, results indicate that the decrease in both PE and AUC by day 1 following treatment is predictive for treatment outcomes at the end of the treatment cycle.

Over the past decade, DCE-US has been increasingly investigated in preclinical [24,26,27] and clinical $[28,29]$ studies to depict therapy induced changes in tumor perfusion. However, a major drawback of conventional DCE-US to date is its limitation to 2D, which results in limited perfusion assessments to a single 2D plane, and results in longitudinal sampling errors. Studies using 2D DCE-US to image breast cancer in mice have in fact demonstrated that slight millimeter-sized deviations in transducer positioning can lead to substantial errors in estimation of tumor perfusion, ranging from $6.4 \%$ to $40.3 \%$ [9]. In clinical studies, inconsistent results have been reported on the use of 2D DCE-US to monitor tumor response during antiangiogenic therapy $[28,30]$. One study found that changes in three perfusion parameters (AUC, Time to Peak [TTP], and slope of wash-in) of 2D DCE-US 15 days after treatment initiation were significantly associated with progression-free survival in patients with advanced hepatocellular carcinoma treated with sorafenib [28]. In contrast, a different study found that perfusion parameters (AUC, TTP and MTT) were not associated with progression-free survival in patients with metastatic renal cell carcinoma treated with sunitinib [30]. In the latter study, the authors concluded that sampling errors were likely contributing to poor reproducibility of the measurements. These further highlight the need for 3D DCE-US for monitoring treatment response [31].

The recent availability of 3D matrix array transducers with volumetric contrast-mode imaging has potentiated 3D DCE-US as a robust technique for assessment of tumor perfusion without sampling errors [10]. A recent study demonstrated that 3D DCE-US allows quantification of early treatment effects [10] and that substantial decreases in tumor perfusion following antiangiogenic treatment can be detected with perfusion parameters $[10,11]$. In addition, quantified perfusion parameters were found to correlate well with ex vivo quantification of vascular densities in histological specimens [10,11]. However, whether these early changes of tissue perfusion parameters from 3D DCE-US could also predict treatment response remained unknown. Furthermore, it remained unknown whether there are dynamic changes of tumor perfusion in both responders and nonresponders during longitudinal antiangiogenic therapy. In this study, the potential of 3D DCE-US for monitoring longitudinal treatment response of antiangiogenic therapy in a more complex animal model set-up was assessed in which two animal models of colon cancer were used to simulate clinical responders and nonresponders. Our results showed that PE and AUC values on 3D DCE-US substantially decreased in the responding tumors 24 hours after treatment initiation, while no significant changes in PE and AUC were noted at any time point in the 
nonresponding tumors. Also, an early decrease of both PE and AUC were highly predictive of later time point treatment response assessed at day 10.

In parallel with DCE-US, 3D ultrasound molecular imaging using molecularly targeted contrast microbubbles is emerging as a promising tool to assess expression levels of angiogenic markers such as VEGFR2 for monitoring antiangiogenic therapy [32]. A recent study has shown that both 3D ultrasound molecular imaging with clinical grade VEGFR2targeted microbubbles and 3D DCE-US with FDA-approved non-targeted microbubbles could provide complementary molecular and functional in vivo information on antiangiogenic treatment effects [11]. Also, early changes in molecular ultrasound imaging signal was highly predictive of later treatment outcomes in another study in a mouse colon cancer xenograft model [33]. Our findings are complementary to these results and demonstrate for the first time that early decrease of PE and AUC values obtained by 3D DCE-US can be predictive of treatment response following several treatment administration cycles. Since the use of ultrasound contrast agents is widely available and approved for clinical use globally, the use of 3D DCE-US for treatment monitoring as described in our study is readily translatable to patients.

Several limitations of this study need to be acknowledged. First, the number of animals in each group was small. However, since strong changes in perfusion parameters were observed in bevacizumab-treated versus saline-treated mice in responding tumors, additional animals were not warranted after statistical analysis. Second, a murine colon cancer model which is known to be refractory to bevacizumab treatment was used to simulate clinical nonresponding tumors. This animal model does not exactly mimic human tumor behavior as tumor resistance often develops during antiangiogenic treatment in patients, besides inherent resistance [3]. Also, subcutaneous colon cancer models may be limited in terms of modeling the tumor microenvironment of metastases in the liver or other organs and longitudinal scanning was limited to 10 days due to rapid tumor growth with outgrowth of control tumors in our model tumors. Therefore, future longer term longitudinal studies in orthotopically implanted cancer or in transgenic mice along with early clinical trials in patients are warranted to confirm the results of this study. Finally, 3D DCE-US imaging results were only compared with the percentage area of neovasculature in this study as bevacizumab has a direct antiangiogenic effect and ultrasound perfusion parameters depend on the number of perfused vessels. Previous studies have demonstrated increased hypoxia following antiangiogenic therapy in human colon cancer xenografts in mice following bevacizumab treatment $[15,18]$; also, bevacizumab has been shown to upregulate apoptosis executioner protein cleaved caspase-3 and to downregulate both antiapoptotic protein Bcl-2 and tumor cell proliferation marker p-Erk expressions in human squamous cell carcinoma xenografts in mice [34].

\section{Conclusions}

In conclusion, our results suggest that 3D DCE-US is sensitive to early tumor response after antiangiogenic treatment, and changes in quantitative parameters are predictive of treatment response in an animal model of human colon cancer. Since 3D DCE-US provides a robust and reproducible longitudinal imaging platform, clinical studies are under way to test 
whether this technique can be clinically translated and allows detection of early treatment changes in patients undergoing antiangiogenic and other therapies.

\section{Acknowledgments}

Funding: This research was supported by the NIH R01 CA155289 grant (JKW) and NIH R01 CA195443 (JKW).

We would like to thank Philips for providing the EPIQ7 US system. We would also like to thank the China Scholarship Council and Program for New Century Excellent Talents in University for providing funding for Dr. Jianhua Zhou to study abroad at Stanford University.

\section{References}

1. Folkman J. Tumor angiogenesis: therapeutic implications. N Engl J Med. 1971; 285(21):1182-1186. DOI: 10.1056/NEJM197111182852108 [PubMed: 4938153]

2. Folkman J. Role of angiogenesis in tumor growth and metastasis. Semin Oncol. 2002; 29(6 Suppl 16):15-18. DOI: $10.1053 /$ sonc.2002.37263

3. Shojaei F. Anti-angiogenesis therapy in cancer: current challenges and future perspectives. Cancer Lett. 2012; 320(2):130-137. DOI: 10.1016/j.canlet.2012.03.008 [PubMed: 22425960]

4. Cremolini C, Loupakis F, Antoniotti C, Lupi C, Sensi E, Lonardi S, Mezi S, Tomasello G, Ronzoni M, Zaniboni A, Tonini G, Carlomagno C, Allegrini G, Chiara S, D’Amico M, Granetto C, Cazzaniga M, Boni L, Fontanini G, Falcone A. FOLFOXIRI plus bevacizumab versus FOLFIRI plus bevacizumab as first-line treatment of patients with metastatic colorectal cancer: updated overall survival and molecular subgroup analyses of the open-label, phase 3 TRIBE study. Lancet Oncol. 2015; 16(13):1306-1315. DOI: 10.1016/S1470-2045(15)00122-9 [PubMed: 26338525]

5. Bennouna J, Sastre J, Arnold D, Osterlund P, Greil R, Van Cutsem E, von Moos R, Vieitez JM, Bouche O, Borg C, Steffens CC, Alonso-Orduna V, Schlichting C, Reyes-Rivera I, Bendahmane B, Andre T, Kubicka S. Investigators MLS. Continuation of bevacizumab after first progression in metastatic colorectal cancer (ML18147): a randomised phase 3 trial. Lancet Oncol. 2013; 14(1):29_ 37. DOI: 10.1016/S1470-2045(12)70477-1 [PubMed: 23168366]

6. Therasse P, Arbuck SG, Eisenhauer EA, Wanders J, Kaplan RS, Rubinstein L, Verweij J, Van Glabbeke M, van Oosterom AT, Christian MC, Gwyther SG. New guidelines to evaluate the response to treatment in solid tumors. European Organization for Research and Treatment of Cancer, National Cancer Institute of the United States, National Cancer Institute of Canada. Journal of the National Cancer Institute. 2000; 92(3):205-216. [PubMed: 10655437]

7. Hlatky L, Hahnfeldt P, Folkman J. Clinical application of antiangiogenic therapy: microvessel density, what it does and doesn't tell us. Journal of the National Cancer Institute. 2002; 94(12):883893. [PubMed: 12072542]

8. Marcus CD, Ladam-Marcus V, Cucu C, Bouche O, Lucas L, Hoeffel C. Imaging techniques to evaluate the response to treatment in oncology: current standards and perspectives. Crit Rev Oncol Hematol. 2009; 72(3):217-238. DOI: 10.1016/j.critrevonc.2008.07.012 [PubMed: 18760935]

9. Hoyt K, Sorace A, Saini R. Quantitative mapping of tumor vascularity using volumetric contrastenhanced ultrasound. Invest Radiol. 2012; 47(3):167-174. DOI: 10.1097/RLI.0b013e318234e6bc [PubMed: 22104962]

10. Wang H, Hristov D, Qin J, Tian L, Willmann JK. Three-dimensional Dynamic Contrast-enhanced US Imaging for Early Antiangiogenic Treatment Assessment in a Mouse Colon Cancer Model. Radiology. 2015; 277(2):424-434. DOI: 10.1148/radiol.2015142824 [PubMed: 26020439]

11. Wang H, Lutz AM, Hristov D, Tian L, Willmann JK. Intra-Animal Comparison between Threedimensional Molecularly Targeted US and Three-dimensional Dynamic Contrast-enhanced US for Early Antiangiogenic Treatment Assessment in Colon Cancer. Radiology. 2017; 282(2):443-452. DOI: 10.1148/radiol.2016160032 [PubMed: 27490690]

12. Varey AH, Rennel ES, Qiu Y, Bevan HS, Perrin RM, Raffy S, Dixon AR, Paraskeva C, Zaccheo O, Hassan AB, Harper SJ, Bates DO. VEGF 165 b, an antiangiogenic VEGF-A isoform, binds and inhibits bevacizumab treatment in experimental colorectal carcinoma: balance of pro- and 
antiangiogenic VEGF-A isoforms has implications for therapy. Br J Cancer. 2008; 98(8):13661379. DOI: 10.1038/sj.bjc.6604308 [PubMed: 18349829]

13. Fischer C, Jonckx B, Mazzone M, Zacchigna S, Loges S, Pattarini L, Chorianopoulos E, Liesenborghs L, Koch M, De Mol M, Autiero M, Wyns S, Plaisance S, Moons L, van Rooijen N, Giacca M, Stassen JM, Dewerchin M, Collen D, Carmeliet P. Anti-PlGF inhibits growth of VEGF(R)-inhibitor-resistant tumors without affecting healthy vessels. Cell. 2007; 131(3):463-475. DOI: 10.1016/j.cell.2007.08.038 [PubMed: 17981115]

14. Xu L, Stevens J, Hilton MB, Seaman S, Conrads TP, Veenstra TD, Logsdon D, Morris H, Swing DA, Patel NL, Kalen J, Haines DC, Zudaire E, St Croix B. COX-2 inhibition potentiates antiangiogenic cancer therapy and prevents metastasis in preclinical models. Sci Transl Med. 2014; 6(242):242ra284.doi: 10.1126/scitranslmed.3008455

15. Ren Y, Fleischmann D, Foygel K, Molvin L, Lutz AM, Koong AC, Jeffrey RB, Tian L, Willmann JK. Antiangiogenic and radiation therapy: early effects on in vivo computed tomography perfusion parameters in human colon cancer xenografts in mice. Invest Radiol. 2012; 47(1):25-32. DOI: 10.1097/RLI.0b013e31823a82f6 [PubMed: 22178893]

16. Heijmen L, Punt CJ, Ter Voert EG, de Geus-Oei LF, Heerschap A, Bussink J, Sweep CG, Zerbi V, Oyen WJ, Span PN, Boerman O, van Laarhoven HW. Monitoring the effects of bevacizumab beyond progression in a murine colorectal cancer model: a functional imaging approach. Investigational new drugs. 2013; 31(4):881-890. DOI: 10.1007/s10637-012-9920-9 [PubMed: 23325291]

17. Zhang HP, Shi QS, Li F, Liu L, Bai M, Gu JY, Wu Y, Du LF. Regions of interest and parameters for the quantitative analysis of contrast-enhanced ultrasound to evaluate the anti-angiogenic effects of bevacizumab. Mol Med Rep. 2013; 8(1):154-160. DOI: 10.3892/mmr.2013.1499 [PubMed: 23722237]

18. Heijmen L, Ter Voert EG, Punt CJ, Heerschap A, Oyen WJ, Bussink J, Sweep CG, Laverman P, Span PN, de Geus-Oei LF, Boerman OC, van Laarhoven HW. Monitoring hypoxia and vasculature during bevacizumab treatment in a murine colorectal cancer model. Contrast media \& molecular imaging. 2014; 9(3):237-245. DOI: 10.1002/cmmi.1564 [PubMed: 24700751]

19. Greis C. Quantitative evaluation of microvascular blood flow by contrast-enhanced ultrasound (CEUS). Clin Hemorheol Microcirc. 2011; 49(1-4):137-149. DOI: 10.3233/CH-2011-1464 [PubMed: 22214685]

20. Schlosser J, Kirmizibayrak C, Shamdasani V, Metz S, Hristov D. Automatic 3D ultrasound calibration for image guided therapy using intramodality image registration. Phys Med Biol. 2013; 58(21):7481-7496. DOI: 10.1088/0031-9155/58/21/7481 [PubMed: 24099806]

21. Heckel F, Schwier M, H-OP. Object oriented application development with MeVisLab and Python. GI Jahrestagung. 2009; 154:1338-1351.

22. Strouthos C, Lampaskis M, Sboros V, McNeilly A, Averkiou M. Indicator dilution models for the quantification of microvascular blood flow with bolus administration of ultrasound contrast agents. IEEE transactions on ultrasonics, ferroelectrics, and frequency control. 2010; 57(6):1296-1310. DOI: 10.1109/TUFFC.2010.1550

23. Quaia E. Assessment of tissue perfusion by contrast-enhanced ultrasound. Eur Radiol. 2011; 21(3): 604-615. DOI: 10.1007/s00330-010-1965-6 [PubMed: 20927527]

24. Wang JW, Zheng W, Liu JB, Chen Y, Cao LH, Luo RZ, Li AH, Zhou JH. Assessment of early tumor response to cytotoxic chemotherapy with dynamic contrast-enhanced ultrasound in human breast cancer xenografts. PLoS One. 2013; 8(3):e58274.doi: 10.1371/journal.pone.0058274 [PubMed: 23469274]

25. Obermueller E, Vosseler S, Fusenig NE, Mueller MM. Cooperative autocrine and paracrine functions of granulocyte colony-stimulating factor and granulocyte-macrophage colonystimulating factor in the progression of skin carcinoma cells. Cancer Res. 2004; 64(21):78017812. DOI: 10.1158/0008-5472.CAN-03-3301 [PubMed: 15520186]

26. Chen Y, Han F, Cao LH, Li C, Wang JW, Li Q, Zheng W, Guo ZX, Li AH, Zhou JH. Doseresponse relationship in cisplatin-treated breast cancer xenografts monitored with dynamic contrast-enhanced ultrasound. BMC Cancer. 2015; 15:136.doi: 10.1186/s12885-015-1170-8 [PubMed: 25884471] 
27. Baetke SC, Rix A, Tranquart F, Schneider R, Lammers T, Kiessling F, Lederle W. Squamous Cell Carcinoma Xenografts: Use of VEGFR2-targeted Microbubbles for Combined Functional and Molecular US to Monitor Antiangiogenic Therapy Effects. Radiology. 2016; 278(2):430-440. DOI: 10.1148/radiol.2015142899 [PubMed: 26313618]

28. Zocco MA, Garcovich M, Lupascu A, Di Stasio E, Roccarina D, Annicchiarico BE, Riccardi L, Ainora ME, Ponziani F, Caracciolo G, Rapaccini GL, Landolfi R, Siciliano M, Pompili M, Gasbarrini A. Early prediction of response to sorafenib in patients with advanced hepatocellular carcinoma: the role of dynamic contrast enhanced ultrasound. J Hepatol. 2013; 59(5):1014-1021. DOI: 10.1016/j.jhep.2013.06.011 [PubMed: 23811306]

29. Hoyt K, Umphrey H, Lockhart M, Robbin M, Forero-Torres A. Ultrasound imaging of breast tumor perfusion and neovascular morphology. Ultrasound Med Biol. 2015; 41(9):2292-2302. DOI: 10.1016/j.ultrasmedbio.2015.04.016 [PubMed: 26116159]

30. Williams R, Hudson JM, Lloyd BA, Sureshkumar AR, Lueck G, Milot L, Atri M, Bjarnason GA, Burns PN. Dynamic microbubble contrast-enhanced US to measure tumor response to targeted therapy: a proposed clinical protocol with results from renal cell carcinoma patients receiving antiangiogenic therapy. Radiology. 2011; 260(2):581-590. DOI: 10.1148/radiol.11101893 [PubMed: 21555352]

31. Lassau N, Bonastre J, Kind M, Vilgrain V, Lacroix J, Cuinet M, Taieb S, Aziza R, Sarran A, Labbe-Devilliers C, Gallix B, Lucidarme O, Ptak Y, Rocher L, Caquot LM, Chagnon S, Marion D, Luciani A, Feutray S, Uzan-Augui J, Coiffier B, Benastou B, Koscielny S. Validation of dynamic contrast-enhanced ultrasound in predicting outcomes of antiangiogenic therapy for solid tumors: the French multicenter support for innovative and expensive techniques study. Invest Radiol. 2014; 49(12):794-800. DOI: 10.1097/RLI.0000000000000085 [PubMed: 24991866]

32. Wang H, Kaneko OF, Tian L, Hristov D, Willmann JK. Three-dimensional ultrasound molecular imaging of angiogenesis in colon cancer using a clinical matrix array ultrasound transducer. Invest Radiol. 2015; 50(5):322-329. DOI: 10.1097/RLI.0000000000000128 [PubMed: 25575176]

33. Zhou J, Wang H, Zhang H, Lutz AM, Tian L, Hristov D, Willmann JK. VEGFR2-Targeted ThreeDimensional Ultrasound Imaging Can Predict Responses to Antiangiogenic Therapy in Preclinical Models of Colon Cancer. Cancer Res. 2016; 76(14):4081-4089. DOI: 10.1158/0008-5472.CAN-15-3271 [PubMed: 27206846]

34. Wang Y, Dong L, Bi Q, Ge X, Zhang X, Wu D, Fu J, Zhang C, Wang C, Li S. Beyond antiangiogenesis: intratumorally injected bevacizumab plays a cisplatin-sensitizing role in squamous cell carcinomas in mice. Chemotherapy. 2011; 57(3):244-252. DOI: 10.1159/000326485 [PubMed: 21597289] 


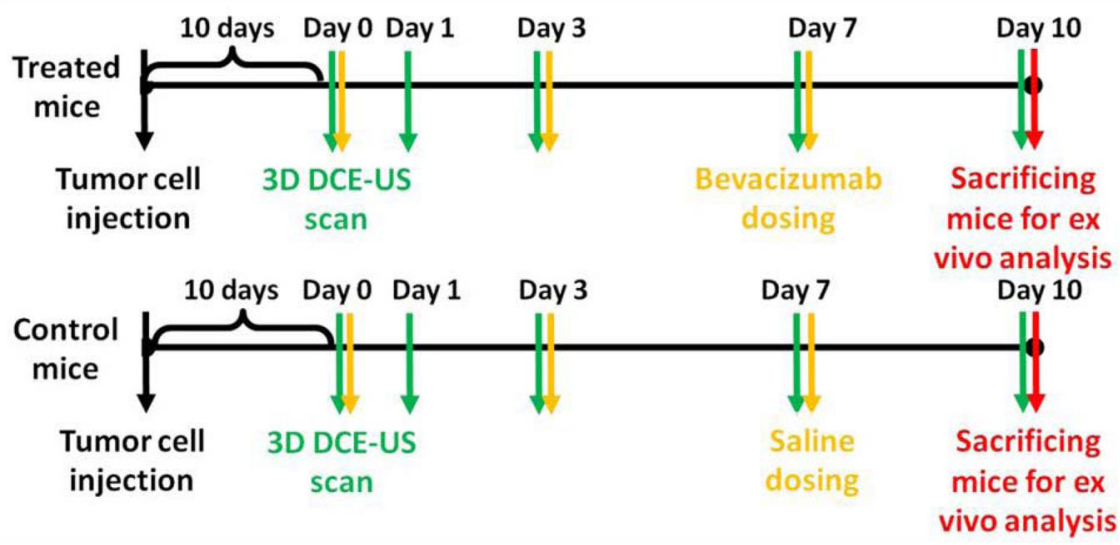

Figure 1.

Summary of experimental study design of 3D DCE-US imaging in both responding (LS174T) and nonresponding (CT26) tumors. Ten days after tumor cell injection, 3D DCEUS scans (green arrows) were performed prior to treatment (baseline scan at day 0), and at subsequent days 1, 3, 7, and 10 after treatment (yellow arrows) using either bevacizumab (treated mice) or saline (control mice). All mice were sacrificed at day 10 (red arrows) and tumors were excised for histologic analysis. 

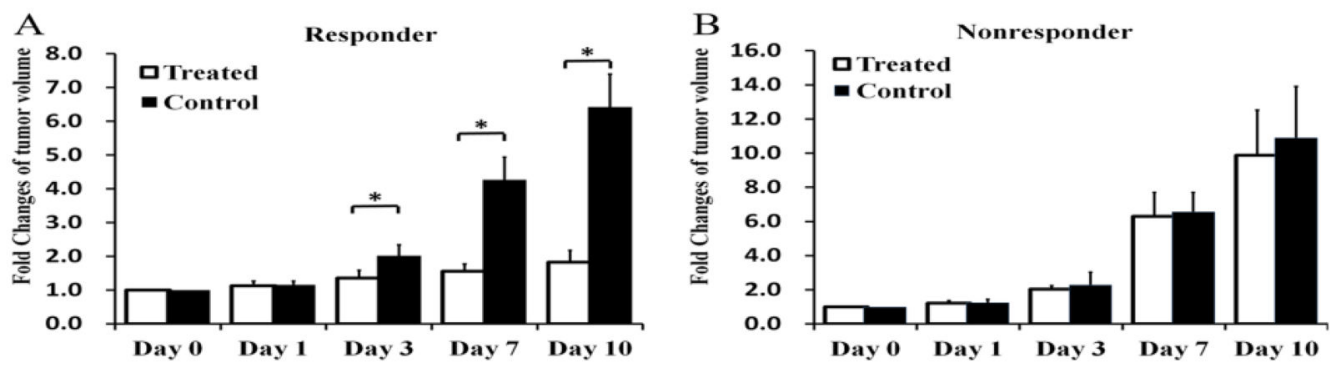

Figure 2.

Bar graphs summarize tumor volumes in responding and nonresponding tumors, either treated with bevacizumab (white) or saline only (black). * $P<0.001$. Bars represent mean values; error bars = standard deviations. 

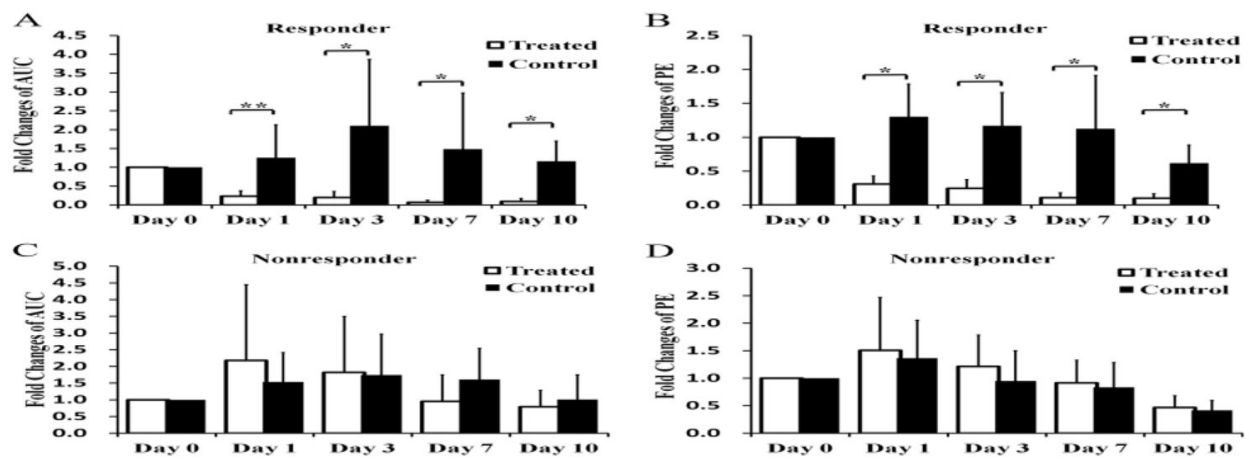

Figure 3.

Bar graph summary of both 3D DCE-US values, AUC (A, C) and PE (B, D), obtained in responding and nonresponding tumors, treated either with bevacizumab (white) or saline only (black). Note that signal decrease in nonresponding tumors and control-treated responding tumors at later time points is likely due to tumor necrosis in these fast growing tumors. * $P<0.001, * * P=0.001$. Bars represent mean values; error bars $=$ standard deviations. 


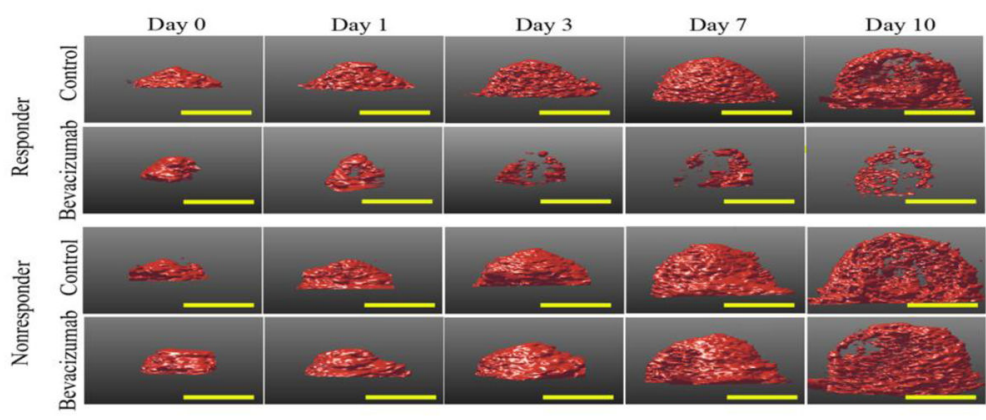

Figure 4.

Representative volume rendered 3D DCE-US imaging data sets at peak enhancement in a responding and nonresponding tumor imaged over a 10-day treatment course. In the responding tumor, 3D DCE-US signal already substantially decreased one day after administration of bevacizumab and remained low during the subsequent days. Note, that while tumor volume slightly increased in responding tumor, 3D DCE-US imaging signal remained low over time. Scale bar $=10 \mathrm{~mm}$. 

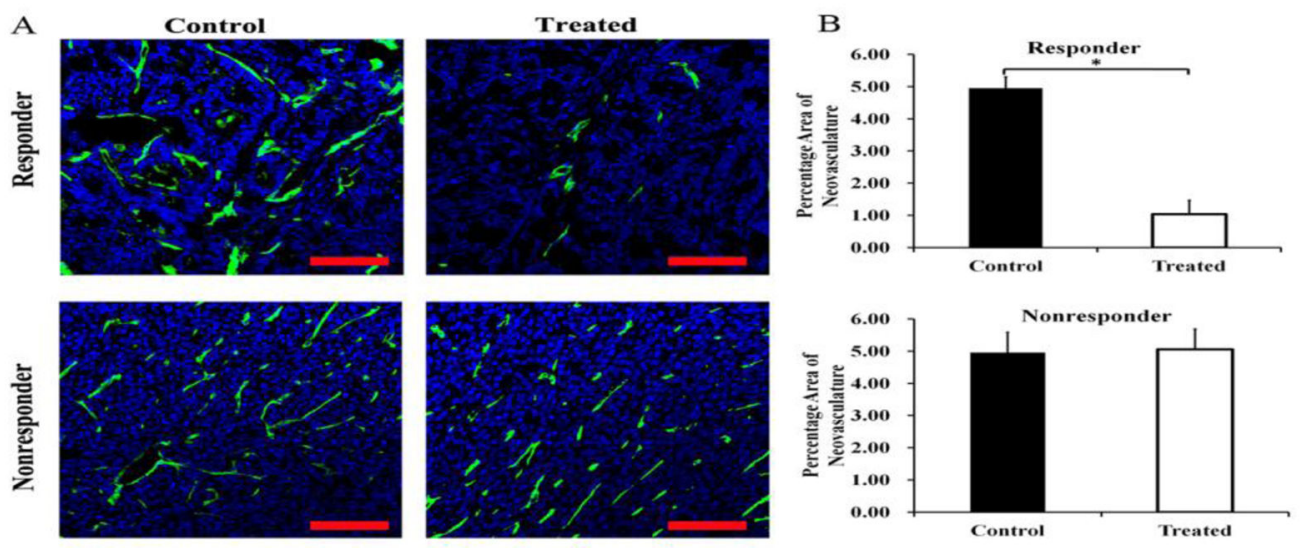

Figure 5.

Immunofluorescence analysis of the tumor neovasculature in responding and nonresponding tumors, either treated with bevacizumab or saline (control). (A) Representative tissue micrographs show tumor neovasculature (green) which substantially decreased in responding tumor compared to nonresponding tumor; blue = DAPI stained cell nuclei. Scale bar $=100 \mu \mathrm{m}$. (B) Bar graphs summarize quantitative results of the measured percentage area of neovasculature. ${ }^{*} P<0.001$. Bars represent mean values; error bars $=$ standard deviations. 